\title{
Insights into CD8 T Cell Activation and Exhaustion from a Mouse Gammaherpesvirus Model
}

\author{
Sally R. Sarawar, Jadon Shen, ${ }^{2}$ and Peter Dias ${ }^{1}$
}

\begin{abstract}
(S.R.S.) I was introduced to viral immunology while working in Peter Doherty's laboratory in the early stages of my research career, inspiring a lifelong interest in this area. During those early years under Peter's mentorship, we studied a mouse gammaherpesvirus model (murine gammaherpesvirus-68 [MHV-68]) that provided a useful small animal model for investigating the immunological control of gammaherpesvirus infection. Interestingly, while CD4 T cells were not required for acute control of MHV-68 in the lung, CD8 T cellmediated control was progressively lost in the absence of CD4 T cell help, leading to viral recrudescence. This was one of several early studies showing that CD8 T cell control of persistent viral infections was lost in the absence of CD4 T cell help, preceding the concept of CD8 T cell exhaustion. Further studies showed that MHV68 infection of mice offered a unique model for comparing the mechanisms of acute and long-term control of a persistent viral infection and developing strategies for reversing $\mathrm{T}$ cell exhaustion. Here, we provide a brief review of the literature on CD8 T cell activation and exhaustion in this model, focusing on the role of CD40 and B7 family members and including some previously unpublished data.
\end{abstract}

Keywords: mouse gammaherpesvirus, CD8 T cell exhaustion, CD40, CD80/86, CD28/CTLA4, PD-1

\section{Introduction}

$\mathbf{M}$ URINE GAMMAHERPESVIRUS-68 (MHV-68) is a naturally occurring rodent pathogen (11) that is closely related to Epstein Barr Virus (EBV) and Kaposi's sarcomaassociated herpesvirus (KSHV) (27,76). EBV and KSHV are associated with a range of human diseases, including infectious mononucleosis, lymphoproliferative disease, nasopharyngeal carcinoma, Kaposi's sarcoma, and lymphoma.

Intranasal administration of $\mathrm{MHV}-68$ to mice results in acute productive infection of lung epithelial cells and a latent infection in various cell types including B lymphocytes, dendritic cells, epithelia, and macrophages (28,29,65,67,73,79). MHV-68 pathogenesis resembles that of EBV in humans, including lymphadenopathy, splenomegaly, and mononucleosis, although structurally the virus is more similar to KSHV $(44,66,68,71,72)$. MHV-68 has also been reported to affect the vasculature, accelerating atherosclerosis in $\mathrm{ApoE}^{-/-}$mice (1) and inducing vasculitis in several types of immunecompromised mice (78).
Infectious MHV-68 is cleared from the lungs by a T celldependent mechanism that can be mediated by either CD4 or CD8 $\mathrm{T}$ cells, while lifelong latency is established in immunocompetent mice $(28,66,69)$. While CD4 $\mathrm{T}$ cells are not essential for primary clearance of replicating virus, they are required for effective long-term control and virus reactivates in the lungs of CD4 T cell-deficient mice, from approximately day 25 after infection onward. This eventually leads to death, probably due to lung damage caused by long-term viral replication $(18,58)$. Similarly, in AIDS patients, CD4 T cell counts are predictive of the incidence and severity of gammaherpesvirusrelated disease, including malignancies (22).

Although an effect of CD4 T cells on CD8 T cell function was initially suspected in the MHV-68 model, several studies failed to find any difference in the number, cytolytic activity, tumor necrosis factor (TNF), or interferon (IFN)- $\gamma$ production of virus specific CD8 T cells from wild-type and CD4 T cell-deficient mice $(7,18,64)$. Furthermore, postexposure vaccination against MHV-68 epitopes known to be protective in a primary response failed to prevent viral

\footnotetext{
${ }^{1}$ Viral Immunology, The Biomedical Research Institute of Southern California, San Diego, California.

${ }^{2}$ Palo Alto Veterans Institute For Research, Palo Alto, California.
}

(C) Sally R. Sarawar et al., 2020; Published by Mary Ann Liebert, Inc. This Open Access article is distributed under the terms of the Creative Commons Attribution Noncommercial License (http://creativecommons.org/licenses/by-nc/4.0/) which permits any noncommercial use, distribution, and reproduction in any medium, provided the original author(s) and the source are cited. 
reactivation, despite massive expansion of virus specific CD8 T cells, suggesting that the inability of CD8 T cells to mediate long-term control of MHV-68 in CD4 T celldeficient mice was not due to a quantitative defect (8).

Closer examination of the expanded $\mathrm{T}$ cell population showed that the relative prevalence of polyfunctional $\mathrm{T}$ cells making both TNF and IFN $\gamma$ and cytotoxic T lymphocyte (CTL) activity on a per cell basis was reduced in spleens of CD4 T celldeficient mice following secondary expansion in vitro $(45,51)$. However, it is unclear how this would affect viral reactivation, when the overall levels of cytokine production or CTL activity were unchanged in $\mathrm{CD} 4 \mathrm{~T}$ cell-deficient mice.

B cell-deficient mice or mice with B cells that are unable to produce virus-specific antibody (77), clear MHV-68 with normal kinetics and do not show viral reactivation in the lungs (65) (S. Sarawar, Unpublished Data). However, T cell depletion during the latent phase of infection, following clearance of infectious MHV-68 in B cell-deficient mice results in viral reactivation (65). Viral reactivation did not occur unless both CD4 and CD8 T cells were depleted, showing that CD8 $\mathrm{T}$ cells could control MHV-68 in the absence of CD4 $\mathrm{T}$ cells, provided that they had been primed in the presence of CD4 T cells (65). These data suggest that CD4 T cells, CD8 T cells, and B cells play overlapping roles in preventing or controlling reactivation of MHV-68 during the latent phase of infection. However, the B and CD8 T cell-mediated control mechanisms do not develop in the absence of an early effect of CD4 T cells.

\section{Costimulatory Molecules in the Immune Response to MHV-68}

Activation of $\mathrm{T}$ cells requires interaction with antigenpresenting cells (APCs). Naïve T cells require two signals for activation-one from the interaction of the $\mathrm{T}$ cell receptor with peptide presented by major histocompatibility complex (MHC) molecules and a second via costimulatory molecules $(35,37,38)$. In some cases a third signal may be required and is often provided by interleukin (IL)-12 (74). However, the sum of multiple positive and negative signals to both the APC and T cells determines the overall response and may change over time in chronic infections.

CD4 T cells are thought to provide "help" in part by conditioning APCs to activate CD8 T cells $(9,19,57,61)$, while there is some evidence that viral infection of APC can bypass this requirement in the generation of CTL (57). This may explain the ability of CD8 T cells to clear some primary viral infections in the absence of CD4 T cell help. However, as exemplified by MHV-68 or lymphocytic choriomeningitis virus (LCMV) infection of mice and human AIDS patients, CD4 $\mathrm{T}$ cells are often required for control of persistent or recurrent viral infections $(18,22,50)$.

\section{$C D 40 / C D 40 L$}

CD40 is expressed on APCs such as dendritic cells, B cells, and macrophages while its ligand is expressed on activated CD4 T cells (48). Stimulation via CD40 on APCs results in upregulation of numerous surface and secreted molecules, initiating cross-talk between APCs and T cells at the immunological synapse.

We showed that stimulation via CD40 could substitute for CD4 $\mathrm{T}$ cell function in the long-term control of MHV-68.
Thus, an agonistic antibody to CD40 prevented reactivation of MHV-68 in MHC Class $\mathrm{II}^{-/-}\left(\mathrm{CII}^{-/-}\right)$mice, which lack CD4 $\mathrm{T}$ cells (59). Injection of the antibody 1 and 15 days after infection had a long-term effect and prevented viral reactivation for at least 100 days (24). In addition, it prevented death in the $\mathrm{CII}^{-/-}$mice. These data confirm that "help" is not required continuously to maintain CD8 T cell function in this model, but appears to be necessary for priming a robust response.

Treatment with an agonistic antibody to CD40 did not appear to stimulate MHV-68-specific antibody, cytolytic activity, or IFN- $\gamma$ production in $\mathrm{CII}^{-/-}$mice, but was ineffective when CD8 TCR+ cells were depleted after initial viral clearance (59). CD8 T cell depleted $\mathrm{CII}^{-/-}$mice showed somewhat higher levels of viral reactivation than control $\mathrm{CII}^{-/-}$mice, suggesting that the "unhelped" CD8 T cells were exerting some level of viral control, although it was inadequate to prevent viral recrudescence $(18,59)$. Since no change in the activity of the CD8 T cells was detected in vitro, a key question was whether the anti-CD40 treatment resulted in a change in the function of the CD8 T cells or of another cell type that was required in addition to CD8 $\mathrm{T}$ cells. To address this question, we showed that adoptive transfer of CD8 T cells from MHV-68 infected wild-type or anti-CD40 treated, but not from control, $\mathrm{CII}^{-/-}$mice caused

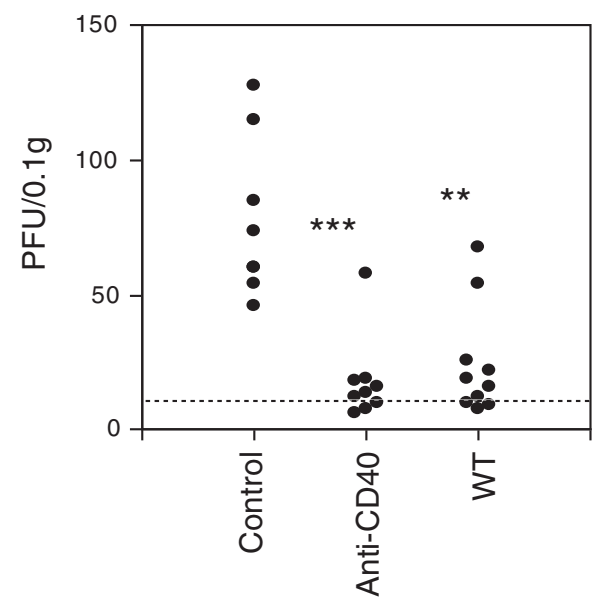

FIG. 1. Anti-CD40 treatment induces a change in CD8 T cell function. Groups of donor or recipient MHC Class $\mathrm{II}^{-/-}$ or donor WT mice were infected with MHV- $68\left(5 \times 10^{4}\right.$ $\mathrm{PFU}$ intranasal). Donor Class $\mathrm{II}^{-/-}$mice were treated with either $100 \mu \mathrm{g} /$ mouse anti-CD40 or isotype control antibody intravenous on days 1 and 15 postinfection. At day 18 after infection, donor mice were killed and CD8 T cells were purified by negative selection from pooled lymph node and spleen cells from each group. $3 \times 10^{6}$ purified CD8 cells were administered intravenously to the recipient mice, which had been lightly irradiated (300 rads) 1 day earlier. Lung virus titers were determined by plaque assay at day 42 after infection. Data are pooled from two independent experiments and represent titers for individual mice. There was a highly significant difference between the lung virus titers in mice that had received cells from control antibodytreated and anti-CD40 treated $\mathrm{CII}^{-1-}$ donors $(* * * p<0.001$, Mann-Whitney Rank Sum test) or WT donors $\left(*^{*} p<0.01\right.$ ). Reprinted with permission from Dias et al. (24). MHV-68, murine gammaherpesvirus-68; WT, wild-type. MHC, major histocompatibility complex; PFU, plaque-forming units. 
a significant reduction in lung virus titers in recipient virusinfected $\mathrm{CII}^{-/-}$(24) (Fig. 1). Thus, anti-CD40 treatment changed CD8 $\mathrm{T}$ cell function, resulting in effective viral control.

Brooks et al. (15) showed, using $\mathrm{CD} 40 \mathrm{~L}^{-1-}$ mice, that CD40L is essential for long-term control of MHV-68. Consistent with these findings, we showed that, like CD4 T cell-deficient mice, $\mathrm{CD} 40^{-1-}$ mice were able to control acute infection with MHV-68, but showed viral reactivation in the lungs at late times after infection (43). Thus, CD40 ligation is both essential and sufficient for long-term control of MHV-68 and there are no redundant pathways.

CD40 is expressed by a number of different cell types including mature dendritic cells and $\mathrm{B}$ cells and activated $\mathrm{T}$ cells. CD40-stimulated dendritic cells or B cells have been shown to act as a "conditioned bridge" mediating $\mathrm{T}$ cell activation in some models $(9,19,57,61)$, whereas others have reported that CD40 expression on CD8 T cells themselves was essential for activation of this cell type (13). Therefore, it was unclear which CD40+ cell type mediated the effect of the agonistic antibody. To address this question, adoptive transfer experiments were performed. Adoptive transfer of antiCD40-treated dendritic cells, but not anti CD40-treated B cells, at days 1 and 15 after infection, significantly reduced lung virus titers in $\mathrm{CII}^{-/-}$mice, although both cell types expressed CD40 and upregulated CD80 and 86 in response to anti-CD40 treatment (34). We observed that the anti-CD40 treatment upregulated CD70 on dendritic cells, but not on $\mathrm{B}$ cells. Athymic nude or $\mathrm{rag}^{-/-}$mice reconstituted with $\mathrm{T}$ cells from $\mathrm{CD}_{40} 0^{+/+}$or $^{-/-}$mice maintained effective longterm control of MHV-68, showing that CD40 on $\mathrm{T}$ cells was not essential for this function, even in the absence of $\mathrm{B}$ cells (34).

\section{CD28}

CD40 stimulation upregulates CD80 (B7-1) and 86 (B7-2) on dendritic cells (19) and these receptors interact with positive and negative costimulatory ligands, CD28 and CD152 (CTLA-4) respectively (4). CD28 is present on resting $\mathrm{T}$ cells and its expression is upregulated following activation. Therefore, it was of interest to determine whether interaction of CD28 with CD80 or CD86 played a role in the effect of CD40 stimulation of dendritic cells on CD8 T cell function in the long-term control of MHV-68. However, two independent studies showed that $\mathrm{CD} 28^{-/-}$mice were able to clear infectious MHV-68 from the lungs and showed effective long-term control of the virus $(42,43)$. Both studies reported that peak infectious virus titers were somewhat higher in $\mathrm{CD} 28^{-1-}$ mice. However, latent virus burden was lower in $\mathrm{CD}^{2} 8^{-1-}$ mice (42).

Although recall interferon gamma responses developed more slowly in CD $28^{-1-}$ mice, CTL activity was similar in $\mathrm{CD} 28^{-/-}$and wild-type mice, consistent with the ability to clear replicating virus from the lungs by day 15 after infection. However, CD $28^{-/}$mice failed to form germinal centers and virus-specific antibody responses were dramatically reduced, with aberrant class switching. Interestingly, Kim et al. (42) showed that the compromised antibody response in CD28 $8^{-1}$ mice was further reduced over time and alone was ineffective in maintaining long-term control of MHV-68. Thus, depletion of both CD4 and CD8 T cells during the latent phase of infection in $\mathrm{CD} 28^{-/-}$mice resulted in viral recrudescence, whereas $\mathrm{T}$ cell depleted wild-type mice could maintain effective viral control. The studies on MHV-68 infection of CD28 $8^{-1-}$ mice also suggested that some CD40-mediated functions in viral control are independent of both CD28 and antibody.

\section{Protein kinase $C$-theta}

Stimulation of $\mathrm{T}$ cells with antigen and CD28 induces translocation of protein kinase C-theta $(\mathrm{PKC} \theta)$, a $\mathrm{T}$ cellspecific isoenzyme of $\mathrm{PKC}$, into the plasma membrane lipid rafts where it co-localizes with the TCR in the immune synapse $(10,52)$. PKC $\theta$ mediates activation of several transcription factors resulting in $\mathrm{T}$ cell activation. We observed that $\mathrm{PKC} \theta^{-1-}$ mice infected with MHV-68 have a similar profile to $\mathrm{CD} 28^{-/-}$mice. Thus, $\mathrm{PKC} \theta^{-1-}$ mice cleared lytic virus and maintained effective long-term control of latency (33). CD8 T cell expansion, recruitment to the lungs and CTL activity were similar in $\mathrm{PKC}^{+/+}$and ${ }^{-/-}$mice, whereas antiviral antibody production and $\mathrm{T}$ helper cell cytokine production were significantly lower in $\mathrm{PKC} \theta^{-/-}$mice (33). This suggested that the pathway by which CD4 T cells act to induce effective longterm control of MHV-68 is not dependent on PKC $\theta$.

Further studies showed that acute control of infectious MHV-68 could be mediated by alternative CD4 T celldependent and PKC $\theta$-dependent pathways of CD8 T cell activation (25). Thus, adoptive transfer of $\mathrm{PKC} \theta^{-/-} \mathrm{CD} 8 \mathrm{~T}$ cells that had developed in the presence of CD4 $\mathrm{T}$ cells reduced lung virus titers in $\mathrm{CD} 4 \mathrm{~T}$ cell-depleted $\mathrm{PKC} \theta^{-/-}$hosts significantly more than the transfer of $\mathrm{PKC} \theta^{-/} \mathrm{CD} 8 \mathrm{~T}$ cells from CD4 $\mathrm{T}$ cell-deficient donors. In addition, CD8 CTL activity from $\mathrm{PKC}^{-1-} \mathrm{CD} 4 \mathrm{~T}$ cell-deficient hosts was dramatically reduced compared with that from $\mathrm{PKC}^{-/-}$control mice. In contrast, CD4 T cell depletion in WT mice did not affect CD8 CTL function. However, long-term control of MHV-68 was dependent on CD4 $\mathrm{T}$ cells, even in PKC $\theta$ sufficient mice $(18,25)$.

\section{$C D 80$ and $C D 86$}

Several studies have reported that the effects of CD80 and CD86 do not always mirror those of CD28 and CTLA4 $(49,81)$ and this is also true in the MHV-68 model. We, and others, reported that $\mathrm{CD} 80 / 86^{-/-}$mice failed to maintain effective long-term control of MHV-68 and showed viral reactivation in the lungs $(32,47)$. However, mice deficient in either CD80 or CD86 alone did not show viral reactivation in the lungs, indicating that these molecules played overlapping roles in the long-term control of MHV-68 (47). Antiviral antibody responses were dramatically reduced in CD80/86 ${ }^{-1-}$ mice, while CD8 CTL activity was not significantly affected $(32,47)$.

IFN- $\gamma$ production by virus-specific CD8 T cells was reduced to a similar extent in $\mathrm{CD} 28^{-1-}$ and $\mathrm{CD} 80 / 86^{-1-}$ mice (32), suggesting that, although this cytokine has been shown to play an important role in the control of latency in MHV68 infection (63), differential production of IFN- $\gamma$ at late time points after infection could not explain the difference in viral reactivation between $\mathrm{CD} 80 / 86^{-/-}$and $\mathrm{CD} 28^{-/-}$mice. Similar to $\mathrm{CD} 28^{-1-}$ mice, CD80/86 $6^{-1-}$ mice showed a reduction in splenic latency in the first few weeks after infection (32). This may relate to the requirement of germinal center 
formation for the establishment of splenic latency (30), as CD28 and CD86 are required for germinal center formation.

Although usually inhibitory, CTLA4 has been reported to also have a positive costimulatory role $(60,80)$. However, Fuse et al. (32) showed that a CTLA4 specific blocking antibody did not induce MHV-68 reactivation in the lungs or increase latent viral load in the spleens of $\mathrm{CD} 28^{-/-}$mice. Furthermore, we demonstrated that CD28/CTLA $4^{-/-}$mice did not show viral reactivation in the lungs (24) (Fig. 2). This ruled out an unusual costimulatory role for CTLA4. Taken together, these data suggest that there could potentially be a novel receptor for CD80 and 86 other than CD28 or CTLA4.

A negative costimulatory interaction between PD-L1 (which also binds to the inhibitory receptor, PD-1) and CD80 has been described $(16,17)$. This was unexpected as both were previously thought to be ligands, although the crystal structure suggests the capability of signaling (16). Our data also argue against a role for CD80-PD-L1 interactions in this model, as the interaction of the proposed novel costimulatory receptor with $\mathrm{CD} 80 / 86$ has a stimulatory effect, resulting in decreased viral titers, while blocking PD-L1 reduced viral reactivation (see below) (24), whereas lack of CD80/86 increases viral titers (47). Furthermore,
Butte et al. (16) reported that PD-L1 only interacts with CD80 and not CD86, whereas CD80 and CD86 have similar functions in our model (47).

If there is a molecule other than CD28 that has a positive costimulatory interaction with CD80 and 86, it has not yet been identified. However, Mandelbrot et al. (49) showed that CD28/CTLA4 ${ }^{-1-} \mathrm{T}$ cell proliferation was inhibited by the use of antigen presenting cells lacking both CD80 and CD86, but could be induced by $\mathrm{CHO}$ cells expressing CD80 or CD86, suggesting that, as in our model, something other than CD28 or CTLA4 provides a positive costimulatory signal via CD80 or CD86.

\section{CTLA4Ig}

CTLA4 binds with higher affinity than CD28 to CD80 and 86 and CTLA4Ig fusion proteins such as belatacept are used clinically to prevent transplant rejection by blocking $\mathrm{T}$ cell costimulation via CD80 and 86. Gammaherpesviruses, such as EBV and KSHV, can present problems in transplant recipients receiving immunosupressive therapy and can cause serious and sometimes fatal post-transplant lymphoproliferative disease (PTLD) or Kaposi's sarcoma. EBV seronegative patients are particularly at risk from primary
A

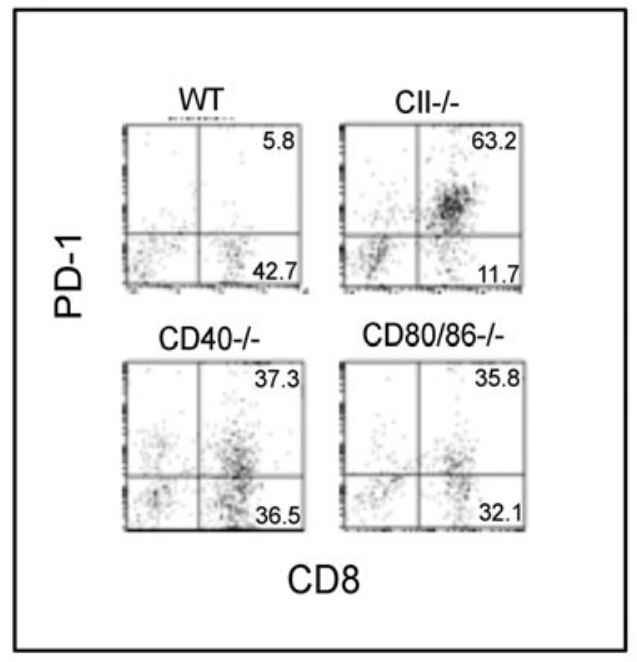

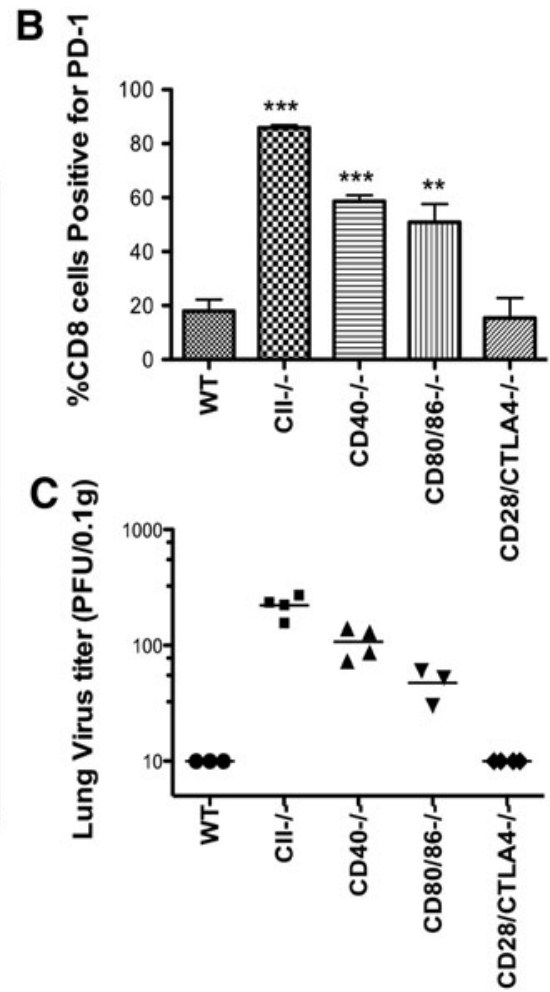

FIG. 2. PD-1 expression on CD8 $\mathrm{T}$ cells and lung virus titers in WT, Class $\mathrm{II}^{-/-}$, CD40 ${ }^{-/-}$, $\mathrm{CD} 80 / 86^{-/-}$, and CD28/ $\mathrm{CTLA}^{-1-}$ mice. Groups of 3-5 mice WT, MHC Class $\mathrm{II}^{-1-}$, CD40 ${ }^{-1-}$, CD80/86 ${ }^{-1-}$, and CD28/CTLA4 ${ }^{-1-}$ mice were infected with MHV-68. Infiltrating cells were harvested from the lung by BAL via the trachea at day 50 postinfection and dualstained with FITC-conjugated anti-CD8 and PE conjugated anti-PD-1. (A). Representative dot plots of PD-1 and CD8 expression in BAL. (B). Mean percentages + standard error of mean of CD8 T cells that were positive for PD-1 in BAL. There was a statistically highly significant difference between the percentage of CD8 T cells positive for PD-1 in the BAL of $\mathrm{CII}^{-1-}, \mathrm{CD} 40^{-/-}$, and CD80/86 ${ }^{-\varphi-}$ mice compared with that of WT mice $* * * p<0.001, * * p<0.01$. There was no significant difference in the percentages for WT and CD28/CTLA $4^{-1-}$ mice. (C). Lung virus titers were determined at day 50 after infection by plaque assay. Data represent titers for individual mice. Reprinted with permission from Dias et al. (24). BAL, bronchoalveolar lavage; FITC, fluorescein isothiocyanate. 
infections acquired from the transplanted tissue, although PTLD also occurs as a consequence of reactivated disease (3). In clinical trials, kidney transplant recipients treated with belatacept (CTLA4Ig) showed a higher incidence of PTLD compared with those treated with cyclosporine, resulting in a black box warning for belatacept $(5,26,75)$.

CTLA4Ig could block T cell costimulatory functions of both $\mathrm{CD} 28$ and the putative novel receptor. The putative novel receptor for CD80 and CD86 could function either at early time points in the generation of a robust immune response or later in the maintenance of the response. To further investigate this, we treated CD28/CTLA4 $4^{-/-}$mice with a noncytolytic form of CTLA4-Ig (which blocks all interactions with CD80 and 86 without depleting cells bearing these molecules) from days 0 to 15,35 to 50 , or 1 to 50 . We were able to provoke viral replication in the lungs with each of these regimens (Fig. 3). This is consistent with the presence of an alternative receptor for CD80 and CD86 and suggests that the putative receptor is important throughout the course of infection.

A study by Pinelli et al. (55) also showed that treatment with CTLA4Ig increased the burden of latent virus in the spleen at day 20 after infection with MHV-68 and reduced the expansion and cytokine production by virus-specific CD8 T cells. Interestingly, rapamycin, which is normally immunosuppressive, was able to reverse the effect on $\mathrm{T}$ cell function and viral burden. Thus, rapamycin increased the number of virus-specific T cells and IFN- $\gamma$ production (55). In a later study, the same group showed that, consistent with studies in CD28 $8^{-/-}$mice, both CTLA4Ig and a CD28-specific antibody decreased the burden of latent virus in the spleen to the same extent at day 14 (probably due to an effect on germinal centers) (21). Titers of infectious virus in the lung were not assessed in either study, as an intraperitoneal route of infection was used, which does not lead to viral replication in the lung.

However, one caveat to studies using CTLA4Ig treatment is that ligation of CD80 and 86 by CTLA4-Ig can trigger indoleamine 2,3 dioxygenase (IDO) activity in dendritic

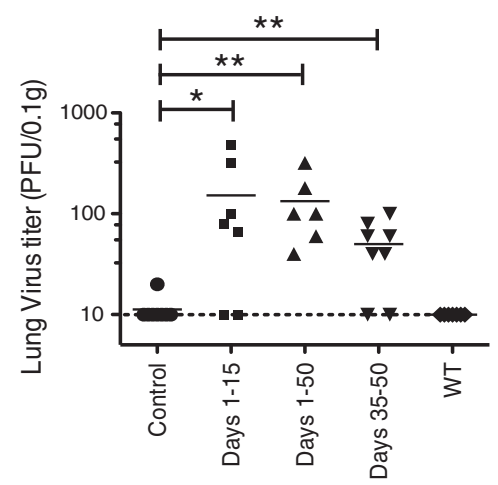

FIG. 3. Viral reactivation induced by CTLA4-Ig in MHV68-infected CD28/CTLA4 ${ }^{--}$mice. Groups of 3-4 CD28/ CTLA4 ${ }^{-/-}$mice were infected with MHV-68 and treated with $0.1 \mathrm{mg} / \mathrm{mouse}$ CTLA4-Ig (Chimerigen) i.p. every 2 days for the indicated time periods. Lung virus titers were determined by plaque assay at day 50 after infection. There was a significant difference between titers in control and CTLA4Ig-treated groups of mice $(* p<0.05, * * p<0.01$, $t$-test). i.p., intraperitoneal; Ig, immunoglobulin. cells by reverse signaling, which allows them to suppress $\mathrm{T}$ cell function $(12,54)$. While initial reports suggested that the effect of IDO was restricted to CD4 T cells, subsequent reports showed that it could also mediate inhibition of CD8 $\mathrm{T}$ cells, during infection with several viruses, including EBV (46). However, T cell suppression by IDO, induced by reverse signaling through CD80 and 86, clearly cannot explain either viral reactivation in $\mathrm{CD} 80 / 86^{-1-}$ mice or the absence of viral reactivation in mice lacking both known ligands for CD80 and 86.

\section{Inhibitory Molecules and T Cell Exhaustion During MHV-68 Infection of Immunocompromised Mice}

Our adoptive transfer experiments showed that there was a qualitative difference in CD8 $\mathrm{T}$ cell from wild-type and $\mathrm{CII}^{-/-}$mice, but it was not clear exactly what that difference was. Therefore, we compared the expression and role of potential inhibitory molecules on the surface of CD8 T cells from $\mathrm{CII}^{-/-}$and wild-type mice (24).

\section{NKG2A}

The heterodimeric molecule NKG2A has been shown to inhibit CD8 $\mathrm{T}$ cell function in a mouse polyoma virus model (53). We noticed a significant reduction in the percentage of CD8 $\mathrm{T}$ cells expressing the inhibitory receptor NKG2A in bronchoalveolar lavage (BAL) from wild-type or antiCD40-treated $\mathrm{CII}^{-1-}$ mice compared to that in control $\mathrm{CII}^{-/-}$ mice (24). In addition, the level of expression was significantly lower in CD8 $\mathrm{T}$ cells from wild-type or anti-CD40 treated $\mathrm{CII}^{-1-}$ mice than in control $\mathrm{CII}^{-1-}$ mice. To address the role of this molecule in the response to MHV-68, we compared the long-term control of MHV-68 in DBA/1 and DBA/2 mice that had been depleted of CD4 T cells. DBA/1 mice are deficient in CD94, which is required for surface expression of NKG2A. However, we saw no difference in the level of viral recrudescence in DBA/1 and DBA/2 mice (24).

\section{$P D-1$ and $P D-L 1$}

The inhibitory molecule PD- 1 is expressed on the surface of CD8 $\mathrm{T}$ cells and has been implicated in CD8 T cell exhaustion in infections with several viruses including LCMV and HIV $(6,20,23,70,82)$. We found that PD-1 expression was significantly upregulated on the surface of CD8 $\mathrm{T}$ cells from Class $\mathrm{II}^{-/-}, \mathrm{CD} 40^{-/-}$, and CD80/86 ${ }^{-/-}$ mice (which all show viral reactivation in the lungs), compared with that on CD8 T cells from either wild-type or CD28/CTLA4 ${ }^{-1-}$ mice (that show effective control of the virus) (Fig. 2) (24). Furthermore, the level of PD-1 expression on CD8 $\mathrm{T}$ cells correlated with the level of viral reactivation in the lungs in the knockout mouse strains, suggesting that PD-1 plays a role in CD8 T cell exhaustion in persistent MHV-68 infection (Fig. 2).

An alternative explanation is that upregulation of PD-1 was a result of viral reactivation, rather than a cause. To distinguish between these possibilities, we blocked PD-1PD-L1 interactions using monoclonal antibodies to either molecule in $\mathrm{CII}^{-/-}$mice. The results showed that blocking PD-1-PD-L1 interactions significantly reduced viral reactivation (Fig. 4) (24). In contrast, antibodies to PD-L2 had no significant effect (24). Blocking both PD-1 and PD-L1 has 
no greater effect than blocking PD-1 alone. Fluorescenceactivated cell sorting analysis of BAL cells from mice treated with anti-PD-1, anti-PD-L1, or anti-PD-L2 showed that the antibodies were nondepleting and that the reduction in viral titers did not significantly reduce the expression of either PD-1 or PD-L1 (24). Thus, upregulation of PD-1 on CD8 T cells in Class $\mathrm{II}^{-/-}$mice appears to be a cause, rather than solely a result, of viral reactivation. However, anti-PD-1 only partially blocked viral reactivation, leading us to postulate that other inhibitory receptors might also be involved.

Freeman et al. (31) showed that PD-1 was upregulated on all MHV-68-specific CD8 T cells, in the absence of CD4 T cell help. CD8 T cells of a subdominant specificity increased in prominence at late times after infection and showed enhanced functionality in that a higher proportion expressed TNF, IFN- $\gamma$, and CD107a, a marker of degranulation. It is possible that these subdominant CD8 $\mathrm{T}$ cells are responsible for some of the residual viral control observed in CD4 T cell-deficient mice, although they are unable to completely block viral reactivation.

\section{Interleukin-10}

Molloy et al. (51) found that levels of IL-10 were higher in the sera of CD4 T cell-depleted MHV-68 infected mice than those from undepleted mice. Similarly, splenocytes from CD4 depleted MHV-68 infected mice produced more IL-10 than those from undepleted mice when restimulated in vitro with MHV-68 peptides. Furthermore, CD4 T celldepleted IL-10R ${ }^{-/-}$mice showed lower levels of MHV-68 reactivation than those from $\mathrm{CD} 4 \mathrm{~T}$ cell-depleted wild-type mice. Using bone marrow chimeras, the authors showed that cell intrinsic production of IL-10 by CD8 T cells compromised long-term control of MHV-68.

\section{Combined effect of blocking IL-10R, PD-1, and TIM-3}

Neither PD-1 nor IL10 blockade alone could completely ablate viral reactivation in $\mathrm{CD} 4 \mathrm{~T}$ cell-deficient mice, leading us to investigate additional inhibitory receptors and the effect of blocking combinations of inhibitory receptors.
A
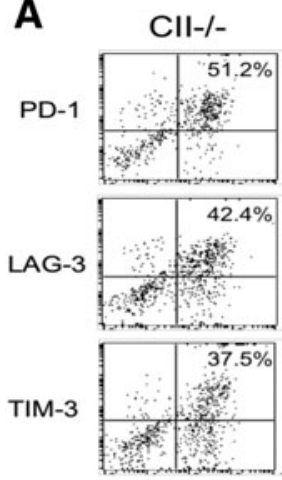

IL-10

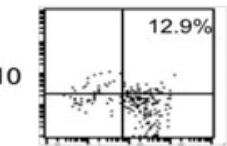

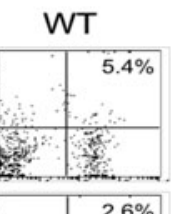

B
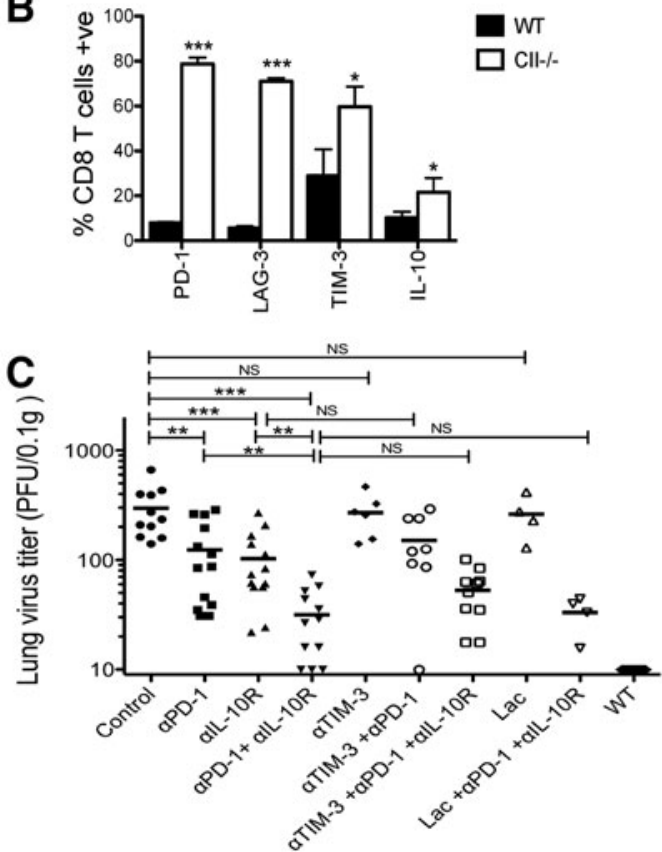

FIG. 4. Increased expression of the inhibitory receptors PD-1, TIM-3, and LAG-3 on the surface of CD8 T cells in the lungs of mice lacking CD4 T cells and effect of blocking inhibitory receptors on lung virus titers. Groups of four $\mathrm{CII}^{-/-} \mathrm{mice}^{-}$ or WT were infected with MHV-68. BAL cells were harvested at day 50 postinfection and dual-stained with FITCconjugated anti-CD8 and phycoerythrin-conjugated anti-PD-1, anti-LAG-3, or anti-TIM-3, followed by flow cytometric analysis. Aliquots were also restimulated with MHV-68 p56 and p79 peptides before surface staining for CD8 and intracellular staining for IL-10. (A) Representative dot plots of PD-1, LAG-3, TIM-3, or IL-10 and CD8 staining. (B) Mean percentages + standard deviation of CD8 T cells that were positive for PD-1, LAG-3, TIM-3, or IL-10 in BAL of WT and MHC Class $\mathrm{II}^{-1-}$ mice. There was a significant difference in the expression of all four molecules between the two groups. $* * * p<0.001, * p<0.05, t$ test. (C). Lung virus titers at day 50 after infection in mice were treated with $0.25 \mathrm{mg} / \mathrm{mouse}$ i.p. of RMP1-14 anti-PD-1, 1B1.3a anti-IL-10R, RMT3-23 anti-TIM-3, combinations of these antibodies, or control rat Ig every 23 days from day 35 postinfection onward. In some groups, $277 \mathrm{mM}$ lactose was added to the drinking water. There was a statistically significant difference between lung viral titers in control Ig and anti-PD-1 $(p<0.01)$, anti-IL-10R ( $p<0.001)$, or anti-PD-1 + anti-IL-10R $(p<0.0001)$ treated Class $\mathrm{II}^{-/}$mice. In contrast there was no significant difference between control and anti-TIM-3 or lactose-treated Class $\mathrm{II}^{-/-}$mice. Class $\mathrm{II}^{-1-}$ mice treated with anti-PD-1 + anti-IL-10R had significantly lower lung viral titers than those treated with anti-PD-1 alone $(p<0.01)$ or anti-IL-10R alone $(p<0.01)$. There was no significant difference in groups treated with anti-PD-1 + anti-IL10R versus anti-PD-1, anti IL-10R + lactose, or antiTIM-3. In addition, there was no significant difference in virus titers in mice treated with anti-TIM-3 + anti-PD-1 versus, those treated with anti-PD-1 alone. ${ }^{*} p<0.01, * * * p<0.001$, NS, not significant. IL, interleukin. 
We found that multiple inhibitory receptors were, in fact, upregulated on the surface of CD8 T cells in CII-deficient mice. In addition to PD-1, LAG-3, and TIM-3 were also upregulated on CD8 $\mathrm{T}$ cells in the BAL of $\mathrm{CII}^{-/-}$versus WT mice (Fig. 4). The majority of exhausted CD8 T cells expressed all three molecules. IL-10 production by CD8 T cells was also modestly increased, consistent with a previous report (51). Recent reports have suggested that the transcription factor, thymocyte selection-associated high mobility group box protein (TOX), is responsible for the transcriptional programming of CD8 $\mathrm{T}$ cell exhaustion $(2,41,62)$ and we have also observed that this transcription factor is expressed at much higher levels in CD8 T cells from $\mathrm{CII}^{-/-}$mice than in those from wild-type mice (L. Lee and S. Sarawar, Unpublished Data).

While blockade of either PD-1 or IL-10R reduced reactivation of MHV-68 in CD4 T cell-deficient mice, combined blockade of both of these molecules had a significantly greater effect than blocking either alone, suggesting that they operate via different mechanisms in this model, as previously reported by Brooks et al. (14) in an LCMV model of T cell exhaustion. In contrast, neither antiTIM-3 nor lactose (which blocks the interaction of TIM-3 with its receptor, galectin 9) had any effect on viral titers, either alone or in combination with anti-IL-10R and antiPD-1. This contrasts with the reported effects of TIM-3 in other viral models of $\mathrm{T}$ cell exhaustion, $(36,39,40,56)$, in which blocking TIM3's interaction with its receptor resulted in improved viral control, highlighting the fact that, while multiple inhibitory receptors are coordinately upregulated on exhausted $\mathrm{T}$ cells in many different viral models, the relative roles of each receptor in suppressing $\mathrm{T}$ cell activity and viral control may vary.

Although LAG3 was also upregulated, it is unlikely that it plays an inhibitory role in the MHV-68 model that we used, as its receptor is MHC Class II, although it could potentially play a role in MHV-68 reactivation in CD4 T cell-depleted wild-type mice. Interestingly, we observed that many of the inhibitory molecules were also upregulated on CD8 T cells during acute infection, when MHV-68 was controlled effectively. Continued expression of inhibitory molecules over several weeks appears to be associated with lack of viral control, although it is not clear whether the same subsets of CD8 T cells are involved at early and late time points.

\section{Summary}

In summary, CD40L on CD4 $\mathrm{T}$ cells activates antigen presenting cells, such as dendritic cells, which then upregulate CD80 and 86 (Fig. 5). CD80 and 86 function in a currently unspecified way that allows programming of a functional change in CD8 T cells, enabling them to maintain effective long-term control of MHV-68. In the absence of CD4 $\mathrm{T}$ cell help, antigen presenting cells do not upregulate CD80 and CD86 and fail to adequately prime CD8 T cells. As a consequence, multiple inhibitory molecules are upregulated on the CD8 T cells by a mechanism that is likely

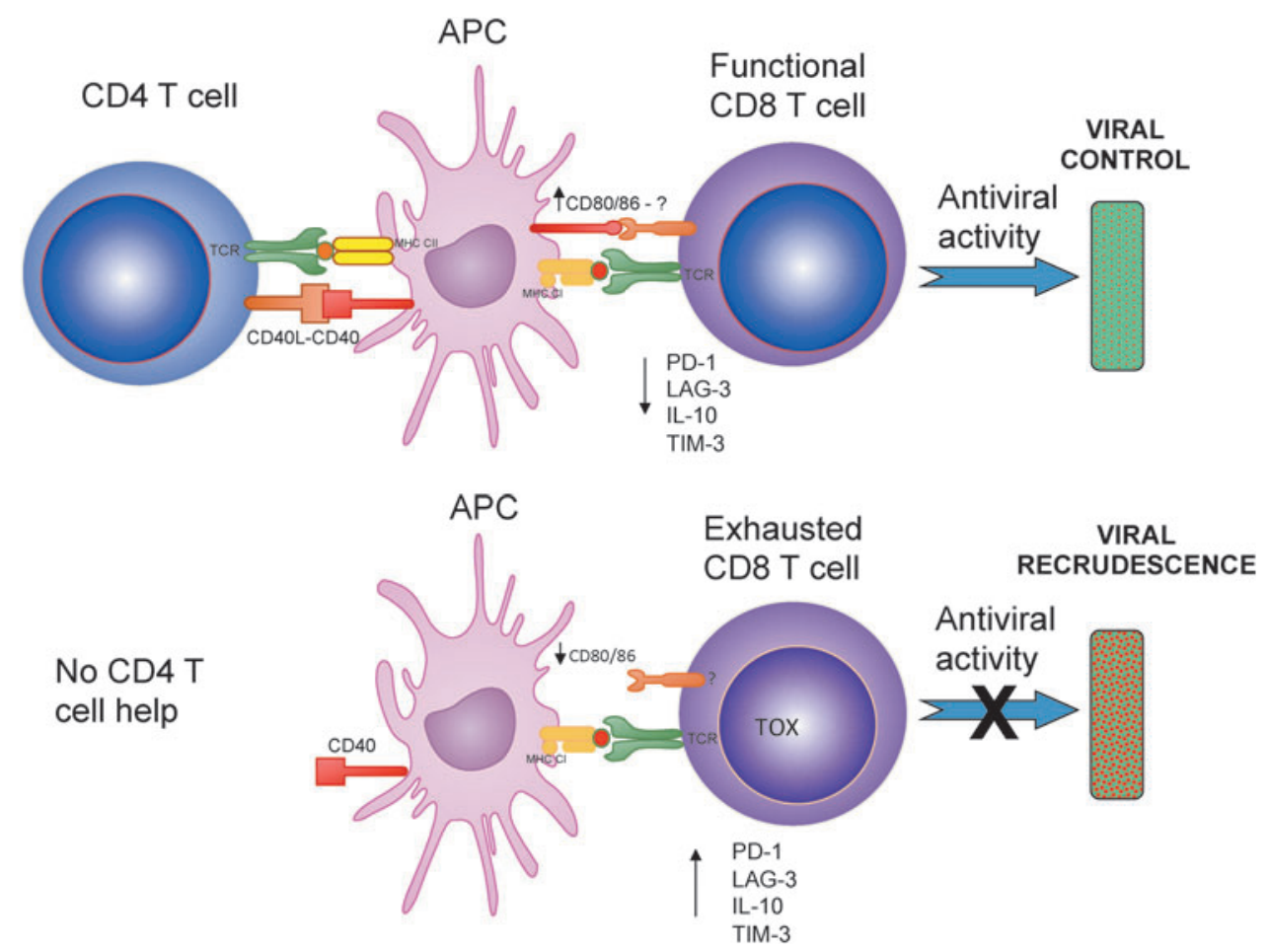

FIG. 5. Model for T cell activation and exhaustion during MHV-68 infection in the presence and absence of CD4 T cell help. CD40L on CD4 T cells activates APCs, which then upregulate CD80 and 86. The activated APCs program a functional change CD8 T cells, enabling them to maintain effective long-term control of MHV-68, by a mechanism that is dependent on CD80/CD86. This change results in reduced expression of multiple inhibitory molecules. In the absence of CD4 T cell help, multiple inhibitory molecules are upregulated by a mechanism that is likely coordinated by TOX. This results in reduced antiviral activity and viral recrudescence. 
coordinated by TOX. This results in reduced antiviral activity and viral recrudescence.

During acute infection, either $\mathrm{PKC} \theta$ or CD4 $\mathrm{T}$ celldependent pathways can mediate viral control, suggesting that the $\mathrm{PKC} \theta$-dependent $\mathrm{CD} 4 \mathrm{~T}$ cell-independent pathway of CD8 $\mathrm{T}$ cell activation was either short-lived or somehow incapable of maintaining long-term control of the virus.

There are several outstanding questions arising from the work described in this review including whether a novel receptor for CD80 and 86 plays a role in viral control, how inhibitory receptors depress CD8 T cell function, what is the precise mechanism of viral control, and whether inhibitory molecules differentially affect CD8 $\mathrm{T}$ cell function in acute and long-term control of MHV-68.

\section{Acknowledgments}

We thank Deanna Leon and Igor Maricic for excellent technical assistance, Dr. Arlene Sharpe for the CD28/ $\mathrm{CTLA}^{-1-}$ mice, and Drs. Hisaya Akiba and Hideo Yagita for providing critical reagents.

\section{Author Disclosure Statement}

No competing financial interests exist.

\section{Funding Information}

We gratefully acknowledge support from the National Institutes of Health grants AI050810 and AI057599.

\section{References}

1. Alber DG, Powell KL, Vallance P, et al. Herpesvirus infection accelerates atherosclerosis in the apolipoprotein E-deficient mouse. Circulation 2000;102:779-785.

2. Alfei F, Kanev K, Hofmann M, et al. TOX reinforces the phenotype and longevity of exhausted T cells in chronic viral infection. Nature 2019;571:265-269.

3. Allen U, Alfieri C, Preiksaitis J, et al. Epstein-Barr virus infection in transplant recipients: summary of a workshop on surveillance, prevention and treatment. Can J Infect Dis 2002;13:89-99.

4. Allison JP. CD28-B7 interactions in T-cell activation. Curr Opin Immunol 1994;6:414-419.

5. Arora S, Tangirala B, Osadchuk L, et al. Belatacept: a new biological agent for maintenance immunosuppression in kidney transplantation. Expert Opin Biol Ther 2012;12: 965-979.

6. Barber DL, Wherry EJ, Masopust D, et al. Restoring function in exhausted CD8 $\mathrm{T}$ cells during chronic viral infection. Nature 2006;439:682-687.

7. Belz GT, Liu H, Andreansky S, et al. Absence of a functional defect in $\mathrm{CD} 8+\mathrm{T}$ cells during primary murine gammaherpesvirus-68 infection of I-A(b-/-) mice. J Gen Virol 2003;84:337-341.

8. Belz GT, Stevenson PG, Castrucci MR, et al. Postexposure vaccination massively increases the prevalence of gammaherpesvirus-specific CD8+ $\mathrm{T}$ cells but confers minimal survival advantage on CD4-deficient mice. Proc Natl Acad Sci U S A 2000;97:2725-2730.

9. Bennett SR, Carbone FR, Karamalis F, et al. Help for cytotoxic-T-cell responses is mediated by CD40 signalling. Nature 1998;393:478-480.
10. Bi K, Tanaka Y, Coudronniere $\mathrm{N}$, et al. Antigen-induced translocation of PKC-theta to membrane rafts is required for T cell activation. Nat Immunol 2001;2:556-563.

11. Blaskovic D, Stancekova M, Svobodova J, et al. Isolation of five strains of herpesviruses from two species of free living small rodents. Acta Virol 1980;24:468.

12. Boasso A, Herbeuval JP, Hardy AW, et al. Regulation of indoleamine 2,3-dioxygenase and tryptophanyl-tRNAsynthetase by CTLA-4-Fc in human CD4+ T cells. Blood 2005;105:1574-1581.

13. Bourgeois C, Rocha B, and Tanchot C. A role for CD40 expression on CD8+ $\mathrm{T}$ cells in the generation of $\mathrm{CD} 8+\mathrm{T}$ cell memory. Science 2002;297:2060-2063.

14. Brooks DG, Ha SJ, Elsaesser H, et al. IL-10 and PD-L1 operate through distinct pathways to suppress T-cell activity during persistent viral infection. Proc Natl Acad Sci U S A 2008;105:20428-20433.

15. Brooks JW, Hamilton-Easton AM, Christensen JP, et al. Requirement for CD40 ligand, CD4(+) T cells, and B cells in an infectious mononucleosis-like syndrome. J Virol 1999;73:9650-9654.

16. Butte MJ, Keir ME, Phamduy TB, et al. Programmed death-1 ligand 1 interacts specifically with the B7-1 costimulatory molecule to inhibit $\mathrm{T}$ cell responses. Immunity 2007;27:111-122.

17. Butte MJ, Pena-Cruz V, Kim MJ, et al. Interaction of human PD-L1 and B7-1. Mol Immunol 2008;45:3567-3572.

18. Cardin RD, Brooks JW, Sarawar SR, et al. Progressive loss of CD8+ T cell-mediated control of a gamma-herpesvirus in the absence of CD4+ T cells. J Exp Med 1996;184:863871.

19. Cella M, Scheidegger D, Palmer-Lehmann K, et al. Ligation of CD40 on dendritic cells triggers production of high levels of interleukin-12 and enhances $\mathrm{T}$ cell stimulatory capacity: T-T help via APC activation. J Exp Med 1996; 184:747-752.

20. Chung EY, Liu J, Homma Y, et al. Interleukin-10 expression in macrophages during phagocytosis of apoptotic cells is mediated by homeodomain proteins Pbx1 and Prep-1. Immunity 2007;27:952-964.

21. Crepeau RL, Elengickal JA, La Muraglia GM, 2nd, et al. Impact of selective CD28 blockade on virus-specific immunity to a murine Epstein-Barr virus homolog. Am J Transplant 2019;19:2199-2209.

22. Crowe SM, Carlin JB, Stewart KI, et al. Predictive value of CD4 lymphocyte numbers for the development of opportunistic infections and malignancies in HIV-infected persons. J Acquir Immune Defic Syndr 1991;4:770-776.

23. Day CL, Kaufmann DE, Kiepiela P, et al. PD-1 expression on HIV-specific T cells is associated with T-cell exhaustion and disease progression. Nature 2006;443:350-354.

24. Dias P, Giannoni F, Lee LN, et al. CD4 T-cell help programs a change in CD8 T-cell function enabling effective long-term control of murine gammaherpesvirus 68: role of PD-1-PD-L1 interactions. J Virol 2010;84:8241-8249.

25. Dias $\mathrm{P}$, Shea AL, Inglis $\mathrm{C}$, et al. Primary clearance of murine gammaherpesvirus 68 by PKCtheta-/- CD8 T cells is compromised in the absence of help from CD4 $\mathrm{T}$ cells. J Virol 2008;82:11970-11975.

26. Durrbach A, Pestana JM, Pearson T, et al. A phase III study of belatacept versus cyclosporine in kidney transplants from extended criteria donors (BENEFIT-EXT study). Am J Transplant 2010;10:547-557. 
27. Efstathiou S, Ho YM, Hall S, et al. Murine herpesvirus 68 is genetically related to the gammaherpesviruses EpsteinBarr virus and herpesvirus saimiri. J Gen Virol 1990;71 (Pt 6):1365-1372.

28. Ehtisham S, Sunil-Chandra NP, and Nash AA. Pathogenesis of murine gammaherpesvirus infection in mice deficient in CD4 and CD8 T cells. J Virol 1993;67:5247-5252.

29. Flano E, Husain SM, Sample JT, et al. Latent murine gamma-herpesvirus infection is established in activated B cells, dendritic cells, and macrophages. J Immunol 2000; 165:1074-1081.

30. Flano E, Kim IJ, Woodland DL, et al. Gamma-herpesvirus latency is preferentially maintained in splenic germinal center and memory B cells. J Exp Med 2002;196:1363-1372.

31. Freeman ML, Roberts AD, Burkum CE, et al. Promotion of a subdominant CD8 $\mathrm{T}$ cell response during murine gammaherpesvirus 68 infection in the absence of CD4 T cell help. J Virol 2014;88:7862-7869.

32. Fuse S, Obar JJ, Bellfy S, et al. CD80 and CD86 control antiviral CD8+ T-cell function and immune surveillance of murine gammaherpesvirus 68. J Virol 2006;80:9159-9170.

33. Giannoni F, Lyon AB, Wareing MD, et al. Protein kinase $\mathrm{C}$ theta is not essential for T-cell-mediated clearance of murine gammaherpesvirus 68. J Virol 2005;79:6808-6813.

34. Giannoni $\mathrm{F}$, Shea A, Inglis $\mathrm{C}$, et al. CD40 engagement on dendritic cells, but not on $\mathrm{B}$ or $\mathrm{T}$ cells, is required for longterm control of murine gammaherpesvirus 68. J Virol 2008; 82:11016-11022.

35. Gilman SC, and Noelle RJ. Molecular mechanisms of costimulation. Agents Actions Suppl 1998;49:15-16.

36. Golden-Mason L, Palmer BE, Kassam N, et al. Negative immune regulator Tim-3 is overexpressed on T cells in hepatitis $\mathrm{C}$ virus infection and its blockade rescues dysfunctional CD4+ and CD8+ T cells. J Virol 2009;83:9122-9130.

37. Janeway CA, Jr., and Bottomly K. Signals and signs for lymphocyte responses. Cell 1994;76:275-285.

38. Jenkins MK. The ups and downs of $\mathrm{T}$ cell costimulation. Immunity 1994;1:443-446.

39. Jin HT, Anderson AC, Tan WG, et al. Cooperation of Tim3 and PD-1 in CD8 T-cell exhaustion during chronic viral infection. Proc Natl Acad Sci U S A 2010;107:1473314738.

40. Jones RB, Ndhlovu LC, Barbour JD, et al. Tim-3 expression defines a novel population of dysfunctional $\mathrm{T}$ cells with highly elevated frequencies in progressive HIV-1 infection. J Exp Med 2008;205:2763-2779.

41. Khan O, Giles JR, McDonald S, et al. TOX transcriptionally and epigenetically programs CD8(+) T cell exhaustion. Nature 2019;571:211-218.

42. Kim IJ, Flano E, Woodland DL, et al. Antibody-mediated control of persistent gamma-herpesvirus infection. J Immunol 2002;168:3958-3964.

43. Lee BJ, Reiter SK, Anderson M, et al. CD28(-/-) mice show defects in cellular and humoral immunity but are able to control infection with murine gammaherpesvirus 68 . J Virol 2002;76:3049-3053.

44. Lee KS, Groshong SD, Cool CD, et al. Murine gammaherpesvirus 68 infection of IFNgamma unresponsive mice: a small animal model for gammaherpesvirus-associated B-cell lymphoproliferative disease. Cancer Res 2009;69: 5481-5489.

45. Liu H, Andreansky S, Diaz G, et al. Reduced functional capacity of CD8+ T cells expanded by post-exposure vacci- nation of gamma-herpesvirus-infected CD4-deficient mice. J Immunol 2002;168:3477-3483.

46. Liu WL, Lin YH, Xiao H, et al. Epstein-Barr virus infection induces indoleamine 2,3-dioxygenase expression in human monocyte-derived macrophages through p38/mitogenactivated protein kinase and NF-kappaB pathways: impairment in T cell functions. J Virol 2014;88:6660-6671.

47. Lyon $A B$, and Sarawar SR. Differential requirement for CD28 and CD80/86 pathways of costimulation in the longterm control of murine gammaherpesvirus-68. Virology 2006;356:50-56.

48. Mackey MF, Barth RJ, Jr., and Noelle RJ. The role of CD40/CD154 interactions in the priming, differentiation, and effector function of helper and cytotoxic $\mathrm{T}$ cells. J Leukoc Biol 1998;63:418-428.

49. Mandelbrot DA, Oosterwegel MA, Shimizu K, et al. B7dependent T-cell costimulation in mice lacking CD28 and CTLA4. J Clin Invest 2001;107:881-887.

50. Matloubian M, Concepcion RJ, and Ahmed R. CD4+ T cells are required to sustain CD8+ cytotoxic T-cell responses during chronic viral infection. J Virol 1994;68:8056-8063.

51. Molloy MJ, Zhang W, and Usherwood EJ. Suppressive CD8+ T cells arise in the absence of CD4 help and compromise control of persistent virus. J Immunol 2011;186: 6218-6226.

52. Monks CR, Kupfer H, Tamir I, et al. Selective modulation of protein kinase C-theta during T-cell activation. Nature 1997;385:83-86.

53. Moser JM, Gibbs J, Jensen PE, et al. CD94-NKG2A receptors regulate antiviral $\mathrm{CD} 8(+) \mathrm{T}$ cell responses. Nat Immunol 2002;3:189-195.

54. Munn DH, Sharma MD, and Mellor AL. Ligation of B7-1/B72 by human $\mathrm{CD} 4+\mathrm{T}$ cells triggers indoleamine 2,3dioxygenase activity in dendritic cells. J Immunol 2004; 172:4100-4110.

55. Pinelli DF, Wakeman BS, Wagener ME, et al. Rapamycin ameliorates the CTLA4-Ig-mediated defect in CD8(+) $\mathrm{T}$ cell immunity during gammaherpesvirus infection. Am J Transplant 2015;15:2576-2587.

56. Reddy PB, Sehrawat S, Suryawanshi A, et al. Influence of galectin-9/Tim-3 interaction on herpes simplex virus-1 latency. J Immunol 2011;187:5745-5755.

57. Ridge JP, Di Rosa F, and Matzinger P. A conditioned dendritic cell can be a temporal bridge between a CD4+ T-helper and a T-killer cell. Nature 1998;393:474-478.

58. Sarawar SR, Cardin RD, Brooks JW, et al. Gamma interferon is not essential for recovery from acute infection with murine gammaherpesvirus 68. J Virol 1997;71:39163921.

59. Sarawar SR, Lee BJ, Reiter SK, et al. Stimulation via CD40 can substitute for CD4 $\mathrm{T}$ cell function in preventing reactivation of a latent herpesvirus. Proc Natl Acad Sci U S A 2001;98:6325-6329.

60. Schneider H, Valk E, da Rocha Dias S, et al. CTLA-4 upregulation of lymphocyte function-associated antigen 1 adhesion and clustering as an alternate basis for coreceptor function. Proc Natl Acad Sci U S A 2005;102:12861-12866.

61. Schoenberger SP, Toes RE, van der Voort EI, et al. T-cell help for cytotoxic T lymphocytes is mediated by CD40CD40L interactions. Nature 1998;393:480-483.

62. Scott AC, Dundar F, Zumbo P, et al. TOX is a critical regulator of tumour-specific $\mathrm{T}$ cell differentiation. Nature 2019;571:270-274. 
63. Steed AL, Barton ES, Tibbetts SA, et al. Gamma interferon blocks gammaherpesvirus reactivation from latency. J Virol 2006;80:192-200.

64. Stevenson PG, Belz GT, Altman JD, et al. Virus-specific $\mathrm{CD} 8(+) \mathrm{T}$ cell numbers are maintained during gammaherpesvirus reactivation in CD4-deficient mice. Proc Natl Acad Sci U S A 1998;95:15565-15570.

65. Stewart JP, Usherwood EJ, Ross A, et al. Lung epithelial cells are a major site of murine gammaherpesvirus persistence. J Exp Med 1998;187:1941-1951.

66. Sunil-Chandra NP, Efstathiou S, Arno J, et al. Virological and pathological features of mice infected with murine gamma-herpesvirus 68. J Gen Virol 1992;73 (Pt 9):23472356.

67. Sunil-Chandra NP, Efstathiou S, and Nash AA. Murine gammaherpesvirus 68 establishes a latent infection in mouse B lymphocytes in vivo. J Gen Virol 1992;73 (Pt 12): 3275-3279.

68. Tarakanova VL, Suarez F, Tibbetts SA, et al. Murine gammaherpesvirus 68 infection is associated with lymphoproliferative disease and lymphoma in BALB beta2 microglobulin-deficient mice. J Virol 2005;79:1466814679.

69. Topham DJ, Cardin RC, Christensen JP, et al. Perforin and Fas in murine gammaherpesvirus-specific CD8(+) T cell control and morbidity. J Gen Virol 2001;82:1971-1981.

70. Trautmann L, Janbazian L, Chomont N, et al. Upregulation of PD-1 expression on HIV-specific CD8+ T cells leads to reversible immune dysfunction. Nat Med 2006;12:11981202.

71. Tripp RA, Hamilton-Easton AM, Cardin RD, et al. Pathogenesis of an infectious mononucleosis-like disease induced by a murine gamma-herpesvirus: role for a viral superantigen? J Exp Med 1997;185:1641-1650.

72. Usherwood EJ, and Nash A. Lymphoproliferative disease induced by murine herpesvirus-68. Lab Anim Sci 1998;48: 344-345.

73. Usherwood EJ, Stewart JP, Robertson K, et al. Absence of splenic latency in murine gammaherpesvirus 68-infected B cell-deficient mice. J Gen Virol 1996;77 (Pt 11):2819_ 2825 .
74. Valenzuela J, Schmidt C, and Mescher M. The roles of IL-12 in providing a third signal for clonal expansion of naive CD8 T cells. J Immunol 2002;169:6842-6849.

75. Vincenti F, Charpentier B, Vanrenterghem Y, et al. A phase III study of belatacept-based immunosuppression regimens versus cyclosporine in renal transplant recipients (BENEFIT study). Am J Transplant 2010;10:535-546.

76. Virgin HWt, Latreille P, Wamsley $\mathrm{P}$, et al. Complete sequence and genomic analysis of murine gammaherpesvirus 68. J Virol 1997;71:5894-5904.

77. Wang H, and Schlomchik MJ. Regulation of autoreactive anti-IgG (rheumatoid factor) B cells in normal and autoimmune mice. Immunol Res 1999;19:259-270.

78. Weck KE, Dal Canto AJ, Gould JD, et al. Murine gammaherpesvirus 68 causes severe large-vessel arteritis in mice lacking interferon-gamma responsiveness: a new model for virus-induced vascular disease. Nat Med 1997;3:13461353.

79. Weck KE, Kim SS, Virgin HI, et al. Macrophages are the major reservoir of latent murine gammaherpesvirus 68 in peritoneal cells. J Virol 1999;73:3273-3283.

80. Wu Y, Guo Y, Huang A, et al. CTLA-4-B7 interaction is sufficient to costimulate T cell clonal expansion. J Exp Med 1997;185:1327-1335.

81. Yamada A, Kishimoto K, Dong VM, et al. CD28independent costimulation of $\mathrm{T}$ cells in alloimmune responses. J Immunol 2001;167:140-146.

82. Zhang JY, Zhang Z, Wang X, et al. PD-1 up-regulation is correlated with HIV-specific memory CD8+ T-cell exhaustion in typical progressors but not in long-term nonprogressors. Blood 2007;109:4671-4678.

Address correspondence to: Dr. Sally R. Sarawar Viral Immunology

The Biomedical Research Institute of Southern California 4225-H Oceanside Boulevard \#273, Oceanside San Diego, CA 92056

E-mail: ssarawar@bri-sc.org 